\title{
MESURE DES FAIBLES DOSES PAR DÉCLIN DE LA FLUORESCENCE DE VERRES RADIOPHOTOLUMINESCENTS
}

\author{
J. BARTHE (*), L. COMMANAY (*), D. BLANC (*) \\ A.-M. CHAPUIS (**), H. FRANÇOIS (**)
}

(Manuscrit reçu le 4 novembre 1973)

\begin{abstract}
RESUME
Nous présentons une nouvelle méthode de lecture des verres radiophotoluminescents utilisés en dosimétrie des rayonnements, largement indépendante des conditions expérimentales de la mesure.

Le verre est excité par de très brèves impulsions de lumière ultraviolette. On détermine pour un intervalle de temps donné la valeur de la constante de temps de la composante exponentielle qui représente au mieux le déclin de la fluorescence.

La relation entre la constante de temps ainsi déterminée et la dose absorbée par le verre suit une loi expérimentale simple. Cette loi est vérifiée pour des doses comprises entre $0,1 \mathrm{mrad}$ et 50 rads.
\end{abstract}

\section{ABSTRACT}

We describe a new method for reading radiophotoluminescent glasses used in dosimetry. The glass sample is excited with very short light pulses. We determine for a chosen time interval the decay time of the exponential component which fits the fluorescence decay.

The experimental relationship between time constant and absorbed dose in glass follows a simple formula proved for doses between $0,1 \mathrm{mrad}$ and 50 rads.

\section{INTRODUCTION}

L'inconvénient majeur des verres radiophotoluminescents utilisés en dosimétrie est leur fluorescence parasite ou "prédose 》 en l'absence de toute irradiation préalable ; la notion de prédose n'est pas une caractéristique

(*) Centre de Physique Atomique et Laboratoire de Biophysique Pharmaceutique, Université Paul Sabatier, Toulouse (France).

(**) Commissariat à l'Energie Atomique, D.Pr S.T.E.P.P.A., BP $\mathrm{n}^{\circ}$ 6, 92260 Fontenay-auxRoses (France).

RADIOPROTECTION, VOL. 9 - $\mathrm{N}^{\circ} 3$ 
physique du verre, mais, au contraire, une caractéristique essentiellement pratique [1] ; elle varie dans des proportions notables suivant le mode de fabrication, la nature et la pureté des divers composants; elle varie également beaucoup d'un échantillon de verre à l'autre, même s'ils proviennent de la même coulée.

Les courbes de déclin de la fluorescence au cours du temps [2, 3] apportent par leur forme des renseignements intéressants sur la dynamique du mécanisme de la fluorescence et de l'effet de mémoire des doses absorbées [4]. Le déclin obtenu après excitation par une impulsion de lumière ultraviolette ayant la forme d'un créneau rectangulaire représente à un coefficient multiplicatif près la densité de probabilité de désexcitation des divers centres au cours du temps. Dans le cas le plus simple où il n'existe qu'une seule sorte de centres à un seul niveau d'excitation à probabilité de désexcitation constante [5], la courbe de déclin est une exponentielle. Dans le cas plus complexe d'une distribution de «n» centres définis comme précédemment, elle forme un déclin à $n$ composantes exponentielles. La constante de temps de chaque exponentielle représente la durée de vie de chaque type de centre excité, l'amplitude qui lui est associée, proportionnelle à leur nombre, est essentiellement représentative de la dose absorbée.

Lorsque le temps d'analyse postérieur à l'excitation devient grand $(>10 \mu \mathrm{s})$, la densité de probabilité de désexcitation devient très faible et la fluorescence prend un caractère aléatoire (photon). Pour obtenir l'allure précise du déclin, il est alors nécessaire de procéder à un échantillonnage statistique sur un grand nombre de mesures.

\section{DISPOSITIF EXPERIMENTAL}

Le dispositif d'enregistrement de la fluorescence se divise en deux parties, une partie optique incluant la source lumineuse et le système de détection, une partie électronique comprenant les systèmes d'échantillonnage, de stockage et de traitement des données (fig. 1).

Les éléments essentiels de la partie optique sont les suivants : focalisation ;

- le verre et son support ;

- le photomultiplicateur de mesure et son système de collection.

L'obtention d'une source d'impulsions de lumière ultraviolette intense ayant de bonnes caractéristiques dynamiques est un problème difficile (fig. 2). Il est en effet nécessaire de disposer d'une impulsion se rapprochant le plus possible d'un créneau parfait et dont la reproductibilité soit bonne au cours du temps. Pour ces différentes raisons, notre choix s'est porté sur le géné- 


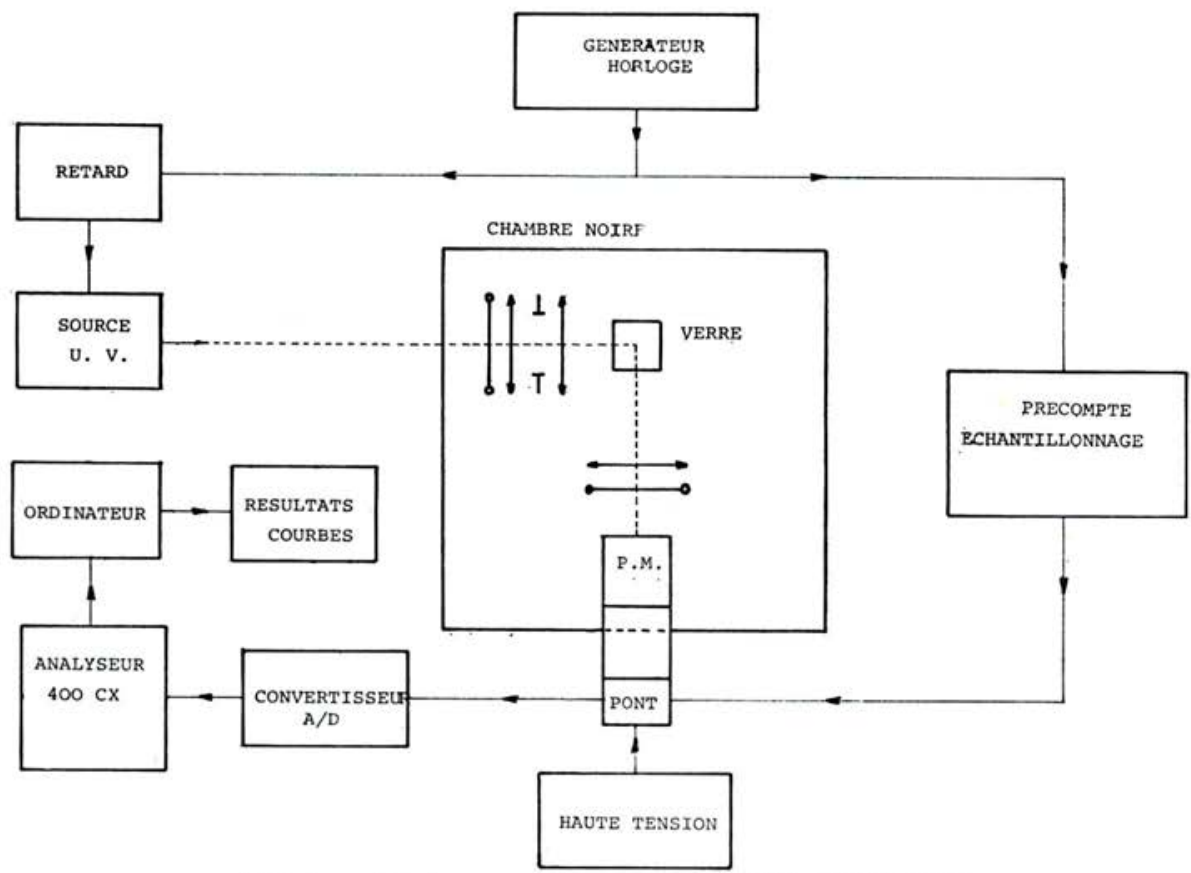

FIG. 1. - Schéma synoptique du dispositif expérimental

rateur «nanoseconde source » de T.R.W. Instruments. Les principales caractéristiques de l'impulsion qu'il fournit sont les suivantes :

- temps de montée $(10$ à $90 \%)<8$ ns ;

— temps de descente $(10$ à $90 \%)<12$ ns ;

- existence d'un fond continu à faible déclin environ $3.10^{-5}$ fors l'intensité crête ;

- fréquence de répétition allant du coup par coup à $5 \mathrm{kHz}$.

La lampe à remplissage d'azote émet essentiellement des raies lumineuses comprises entre 300 et $400 \mathrm{~nm}$ (fig. 3). La sélection de raies particulières est faite à l'aide de filtres interférentiels. L'excitation et la mesure sont effectuées à angle droit afin de séparer au maximum la fluorescence orange de la composante ultraviolette.

La fluorescence est détectée par un photomultiplicateur 56 TVP de la Radiotechnique dont la photocathode de type S20 est particulièrement sensible dans le rouge. Pour éviter un trop grand bruit de fond thermique inhérent à son type de photocathode, il est activement réfroidi $\left(-20^{\circ} \mathrm{C}\right)$.

Les éléments essentiels de la partie électronique sont les suivants :

- le générateur horloge et le circuit d'échantillonnage ;

vol. $9-\mathrm{N}^{\circ} 3$ 


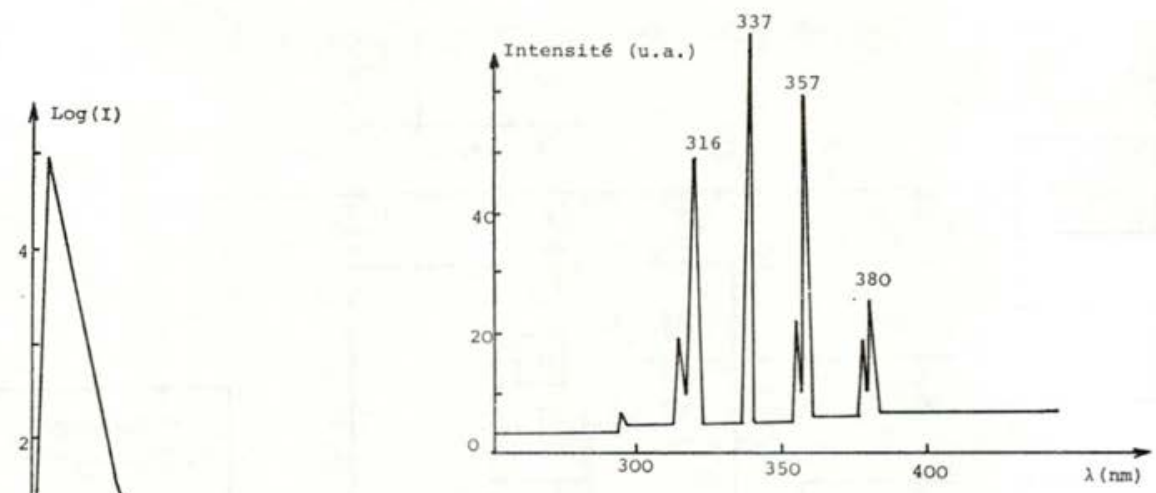

FIG. 3. - Spectre de la lampe à azote digital ;

- la porte d'échantillonnage (P.M.) avec le convertisseur analogique

- une mémoire rapide avec visualisation (analyseur multicanaux (SA 40B) en ligne sur un ordinateur Hewlett Packard (Mod. 20).

Le générateur horloge déclenche successivement, en fonction des retards propres aux différents circuits et du temps de latence choisi, le circuit d'échantillonnage et la source de lumière. La porte d'échantillonnage linéaire est constituée par le photomultiplicateur [6]. Le courant qu'il fournit est intégré sur une capacité $(1000 \mathrm{pF})$, la tension résultante est codée en binaire par un convertisseur analogique digital à 10 bits (Analog Devices) dont la hauteur de marche est $10 \mathrm{mV}$. Cette information numérique est ensuite stockée dans les mémoires de l'analyseur.

Pour restituer la forme de l'impulsion originale, on prend un certain nombre d'échantillons équidistants (généralement 100) couvrant tout le domaine de mesure choisi; ceci constitue un cycle d'échantillonnage. Après un nombre programmé de cycles, le contenu des mémoires est automatiquement transféré dans l'ordinateur. Le nombre de cycles est choisi en fonction de la résolution désirée ; plus ce nombre est grand et meilleure est la résolution. Dès lors peut commencer le traitement des données sous forme d'analyse numérique.

\section{MÉTHODES NUMERIQUES UTILISEEES}

La fonction numérique $I(t)$, intensité de la fluorescence en fonction du temps, obtenue en fin de chaîne de mesure, n'est pas le reflet exact du déclin « pur » de la fluorescence. Les raisons en sont les suivantes : 
Il existe tout d'abord une première convolution d'origine optique entre la véritable forme du déclin de la fluorescence et la forme réelle de l'impulsion d'excitation. En effet, il n'est possible d'obtenir la forme exacte du déclin que par excitation avec un créneau parfaitement rectangulaire (durée nulle du front de descente), ce qui ne peut jamais être réalisé dans la pratique. Il y a donc toujours une excitation partielle de la fluorescence durant la désexcitation. Cela se traduit par la relation suivante :

$$
I(t)=K \int_{0}^{t} P\left(t, t^{\prime}\right) \cdot \phi\left(t^{\prime}\right) \cdot \mathrm{d} t^{\prime}
$$

où $I(t)$ est l'intensité de la fluorescence mesurée ;

$K$ un facteur de proportionnalité ;

$P\left(t, t^{\prime}\right)$ la forme exacte du déclin exprimée sous forme de densité de probabilité, c'est-à-dire la probabilité pour qu'un centre de fluorescence excité à l'instant $t^{\prime}$ 'se désexcite à l'instant $t$;

$\phi\left(t^{\prime}\right)$ la forme de l'impulsion ultraviolette d'excitation.

Ce problème est généralement insoluble, car $P\left(t, t^{\prime}\right)$ est une matrice carrée d'ordre $n, n$ étant le nombre de points de mesure. Il peut toutefois se résoudre dans le cas où $t$ et $t^{\prime}$ interviennent sous la forme d'une différence de temps $t, t^{\prime} \equiv t-t^{\prime}$, ce qui est le cas ici. Dans ces conditions le changement de variable $u=t-t^{\prime}$ conduit à la relation suivante qui n'est autre que l'équation de convolution de Borel :

$$
I(t)=\int_{0}^{t} P(u) \cdot \phi(t-u) \cdot \mathrm{d} u
$$

Les méthodes qui permettent de résoudre cette équation sont peu nombreuses et toujours délicates à mettre en œuvre. Cependant, la simple transformation de l'intégrale continue $\int$ en une somme discrète $\Sigma$ permet dans ce cas précis une solution satisfaisante :

$$
I(t)=\sum_{I=0}^{N} P(I) \cdot \phi(N-I) \cdot h
$$

où $N$ est l'ordre du point $t$;

$h$ l'intervalle de temps entre deux points successifs ;

$I$ une variable entière muette comprise entre 0 et $N$ (limites incluses). En faisant varier $N$ de 0 au nombre maximal de points expérimentaux, on est conduit à résoudre une matrice triangulaire qui ne pose aucune difficulté. 


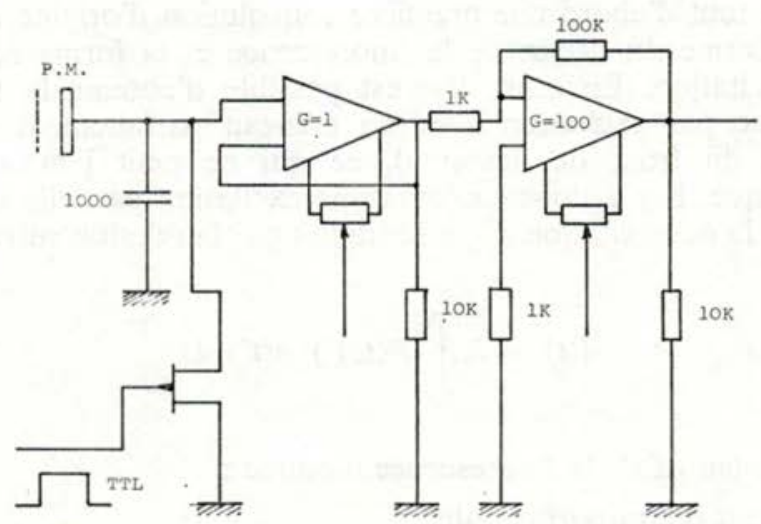

FIG. 4. - Circuit d'anode du photomultiplicateur

Il existe une deuxième convolution d'origine électronique entre la forme effective de l'impulsion de fluorescence et la forme analysée par le convertisseur. Pour améliorer la sensibilité du détecteur, on est amené à intégrer durant un temps déterminé $\varepsilon$ tout le signal fourni par le photomultiplicateur (fig. 4). La tension $V$ aux bornes de la capacité est la suivante :

$$
V(t)=\frac{1}{C} \int_{t}^{t+\varepsilon} I(u) \cdot \mathrm{d} u
$$

où $C$ est la valeur de la capacité ;

$t$ l'instant initial de l'intégration ;

$\varepsilon$ la largeur d'intégration ;

$u$ une variable muette.

Plus $\varepsilon$ sera petit et plus $V(t)$ se confondra avec $I(t)$; toutefois étant donné l'aspect quantique de la lumière recueillie, $\varepsilon$ ne peut pas être indéfiniment petit, car alors la sensibilité du détecteur serait nulle.

Ce système permet de mesurer des débits de fluence compris entre 0 et $10^{10}$ photons/seconde. Il possède le double avantage de l'échantillonnage probabiliste aux faibles débits (photo-électron unique) et linéaire aux forts débits (conversion analogique digitale).

L'analyse numérique de l'équation précédente est facilement soluble si l'on possède une valeur $I(0)$ de référence. En différenciant par rapport à $t$ sous le signe somme on obtient :

$$
V^{\prime}(t)=\frac{1}{C}|I(t+\varepsilon)-I(t)|
$$


Dans le cas où $\varepsilon$ est un multiple entier du pas de mesure sur $t$, on peut écrire :

$$
V^{\prime}\left(t_{i}\right)=\frac{1}{C}\left|I\left(t_{j}\right)-I\left(t_{i}\right)\right| \quad \text { avec } i<j
$$

Ces analyses numériques rentrent dans le cadre du traitement des données.

\section{RESULTATS EXPERIMENTAUX}

Les verres étudiés de composition référencée 33 sont fabriqués par DESMARQuest et C.E.C. Ils sont de deux formes: cubique $(10 \mathrm{~mm})$ et cylindrique $(\varnothing 6 \times 8 \mathrm{~mm})$. Ils ont été stockés environ 8 mois sans condition particulière de protection puis irradiés par une source de ${ }^{60} \mathrm{Co}$. De nombreuses précautions de propreté ont été prises avant la mesure de la fluorescence, notamment un nettoyage actif (RBS 25), un rinçage efficace $\left(10 \mathrm{mn}\right.$ à l'eau désionisée) et un séchage poussé $\left(60^{\circ} \mathrm{C}\right.$ dans une étuve sous vide).

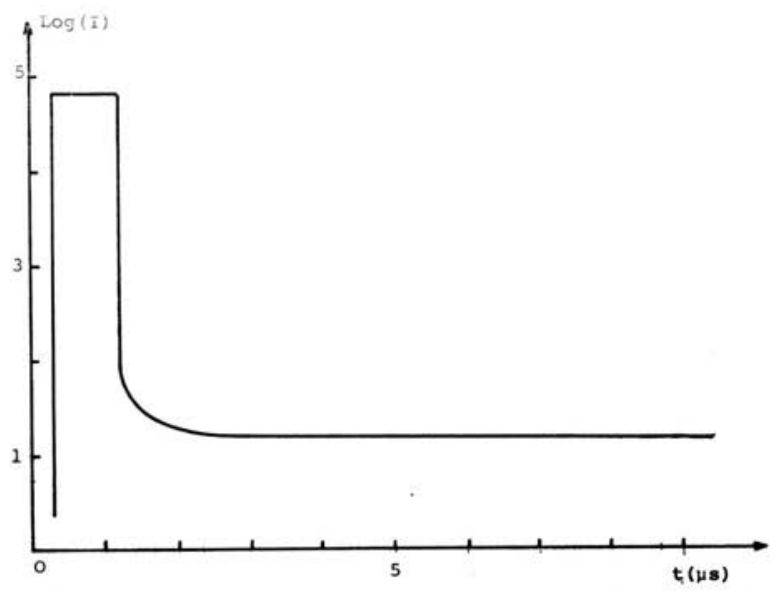

FIG. 5. - Impulsion de lumière vue à travers le circuit d'échantillonnage

Avant de procéder à la mesure de la fluorescence des verres, il est nécessaire de parfaitement connaître, dans les mêmes conditions expérimentales, la forme de l'impulsion de lumière excitatrice. On a pour cela procédé des deux façons suivantes : l'utilisation d'un scintillateur plastique (convertisseur de longueurs d'onde) de constante de temps très courte ( $<$ nanoseconde) devant la variation du signal lumineux et l'utilisation d'un miroir dépoli (faible réflectance) non fluorescent aux rayons ultraviolets. Les deux réponses obtenues (fig. 5) sont parfaitement semblables.

voL. $9-\mathrm{N}^{\circ} 3$ 
La forme d'un déclin de type exponentiel (probabilité de désexcitation constante) est toujours la même quelle que soit l'intensité d'excitation. Ce comportement est vérifié dans le cas des verres étudiés pour différentes doses ( 0 à $10 \mathrm{krad}$ ) et différentes puissances d'excitation (quelques $\mathrm{mW}$ à $1 \mathrm{MW})[3,4]$.

Les verres ont été excités avec plusieurs longueurs d'onde, mais, étant donné la sensibilité globale du système, les mesures ont surtout été faites en spectre total ( $\lambda 300$ à $400 \mathrm{~nm}$ ).

Les travaux antérieurs [3,7] avaient montré la bonne simulation des courbes de déclin par une décomposition en 3 composantes exponentielles; les valeurs sont les suivantes :

constante courte $\quad 0,7 \mu \mathrm{s}$

constante moyenne $2 \rightarrow 3 \mu$ s

constante longue $5 \rightarrow 40 \mu$ s.

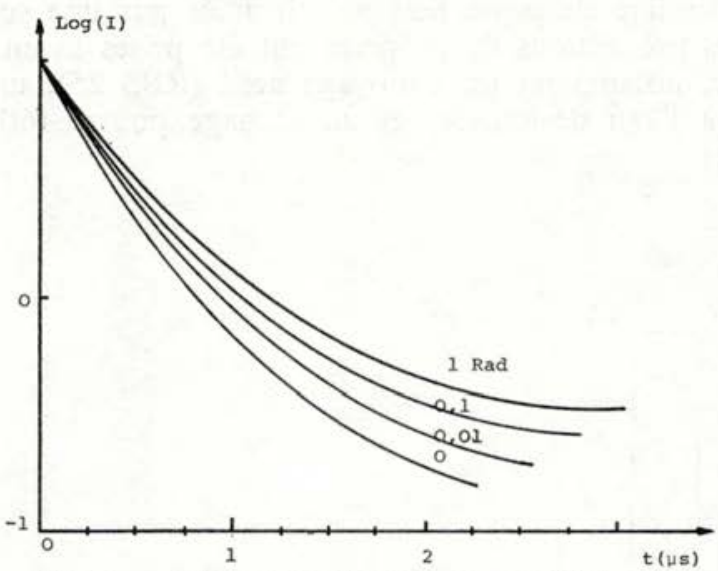

FIG. 6. - Allure de la fluorescence aux faibles doses

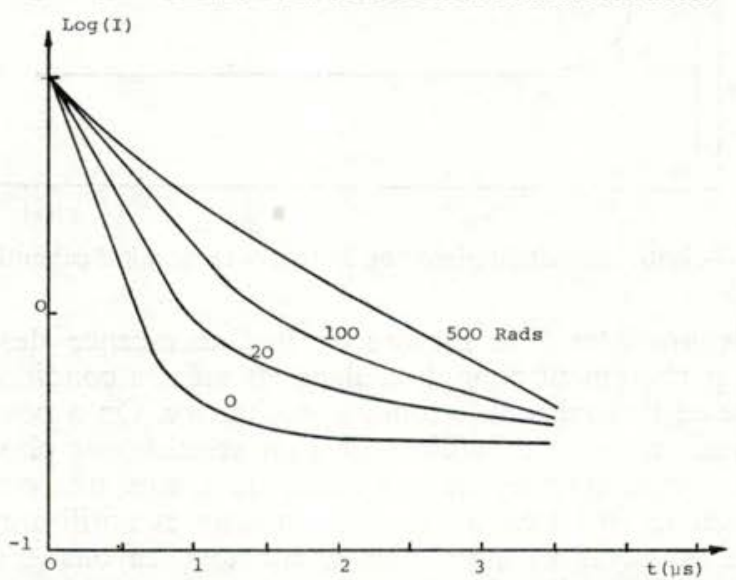

FIg. 7. - Allure de la fluorescence aux fortes doses 
Cependant la grande instabilité de cette analyse numérique conduit à lui préférer la méthode des domaines. Cette méthode consiste à rechercher la constante de temps de la composante exponentielle la plus représentative de la courbe expérimentale dans trois domaines préalablement définis en fonction des résultats précédents. Les domaines choisis sont les suivants :

$$
\begin{aligned}
& \text { domaine } 1: 0 \text { à } 2 \mu \mathrm{s} \\
& \text { domaine } 2: 2 \text { à } 4 \mu \mathrm{s} \\
& \text { domaine } 3: 4 \text { à } 9 \mu \mathrm{s} .
\end{aligned}
$$

Ce choix peut sembler arbitraire et dénué de tout fondement physique. Cependant cette méthode présente bien moins de dispersion. La valeur trouvée, à l'inverse de la constante de temps propre, est influencée par les autres composantes existantes. Elle est toutefois bien adaptée à la détermination de profils d'évolution lorsque le nombre de composantes est grand (supérieur à 4 dans la pratique).

Les figures 6 et 7 montrent les variations de la forme du déclin de la fluorescence (amplitudes normalisées à 10) pour différentes doses. On constate qu'au-delà de 500 rads les courbes sont identiques, il n'y a alors plus de variation de la forme du déclin.

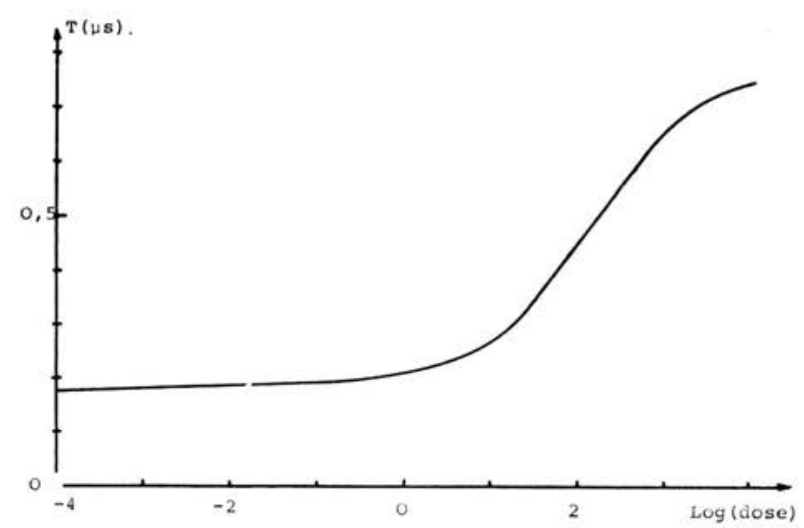

FIG. 8. - Evolution de la constante de temps du domaine 1

Les figures 8 , 9 et 10 montrent respectivement l'évolution de la constante de temps des domaines 1,2 et 3 .

Il apparaît une opposition des évolutions entre les domaines 1, d'une part, et 2, 3, d'autre part, ainsi qu'une similitude entre les domaines 2 et 3. Cela provient essentiellement de l'influence des divers domaines entre eux, influence occasionnée simultanément par les variations de constante de temps et d'amplitude. Toutefois, on retrouve les valeurs de constante de temps énoncées dans les travaux précédents $[3,7]$; on trouve environ $0,7 \mu \mathrm{s}$ (domaine 1), 2,5 à $3,5 \mu$ s (domaine 2) et 5 à $30 \mu$ s (domaine 3) pour des doses inférieures à 50 rads. Les valeurs limites trouvées sont légèrement différentes. 


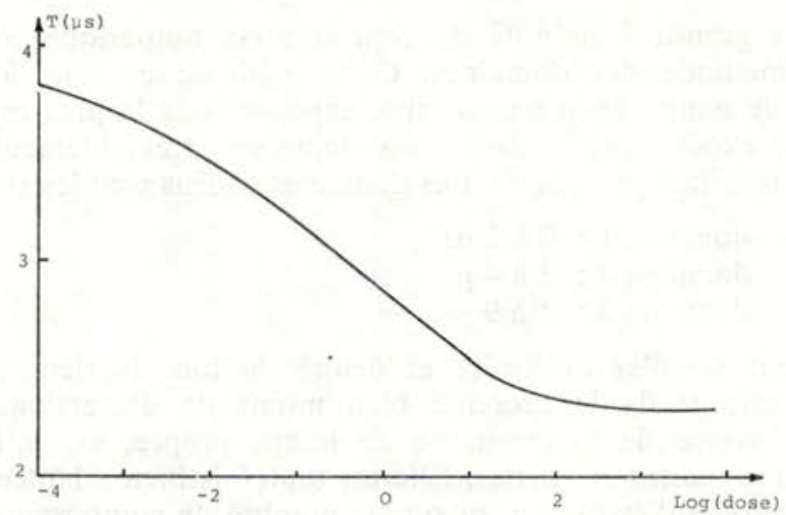

FIG. 9. - Evolution de la constante de temps du domaine 2

Le domaine 3 est le plus intéressant, car l'évolution de la constante de temps semble se faire selon une loi simple (loi linéaire en échelle semilogarithmique) entre $100 \mu \mathrm{rad}$ et 50 rads :

$$
D=D_{0} \cdot \exp \left\{-\frac{T}{T_{\alpha}}\right\}
$$

où $D_{0}=370$ rads (dose extrapolée à $T=0$ );

$T_{\alpha}=1,95 \mu$ s (opposé de la pente exponentielle) ;

D la dose pour la valeur $T$ de la constante de temps.

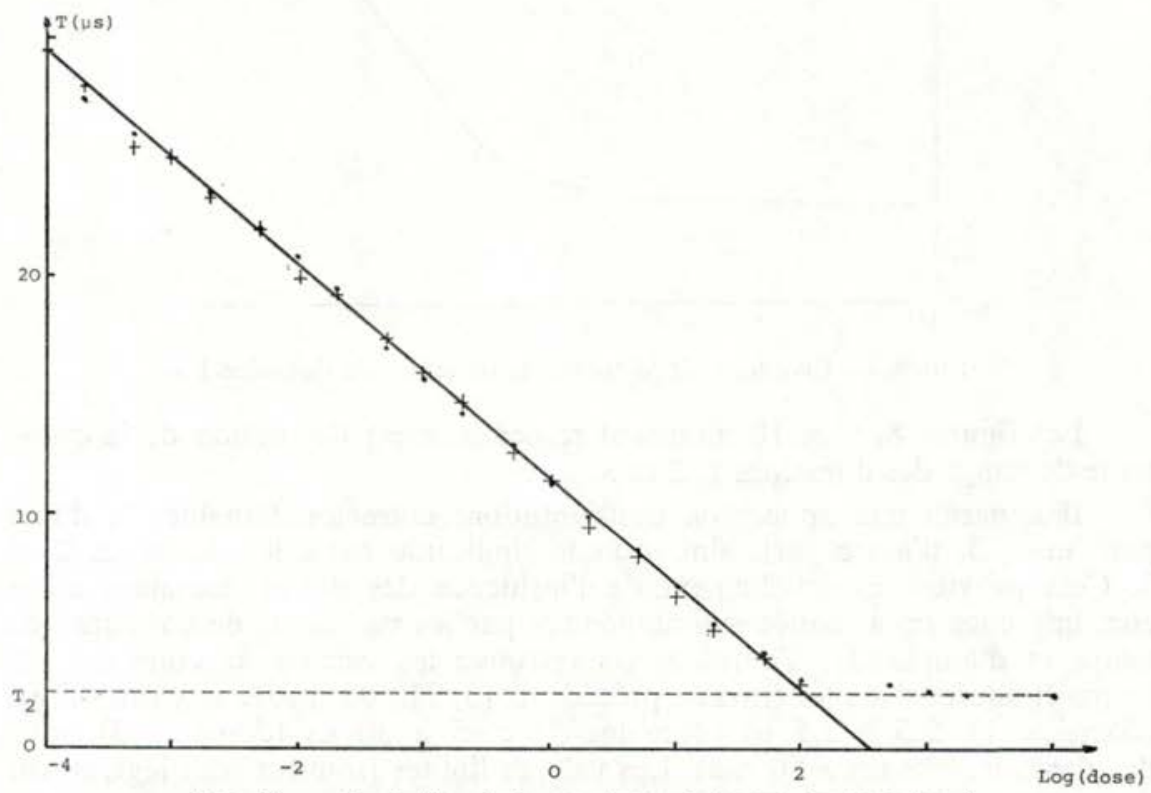

FIG. 10. - Evolution de la constante de temps du domaine 3 
Au-delà de 50 rads la forme de déclin se fige, cela se traduisant par une variation asymptotique des courbes d'évolution. Il est possible d'expliquer une telle allure de courbe en supposant qu'il existe au départ dans le verre une certaine distribution de centres fluorescents $f(\tau), \tau$ étant leur durée de vie. Seule une fraction de cette distribution croît avec la dose. Il apparaîtra dès lors une évolution de la forme de déclin asymptotique à la réponse de cette fraction, ce qui est effectivement observé.

L'extrapolation linéaire aux faibles doses de l'évolution du domaine 3 conduit à penser que les verres vierges dont les constantes de temps sont comprises entre 34,5 et $37,5 \mu$ s correspondent à des prédoses variant de 1,8 à $8 \mu \mathrm{rad}$. Cette dernière constatation est en contradiction avec les valeurs communément admises du rayonnement ambiant naturel $(200 \mathrm{mrad} / \mathrm{an})$.

Ceci pose le problème de l'influence du débit de dose sur la réponse du verre ; les plus faibles irradiations $(100 \mu \mathrm{rad})$ ont été effectuées avec des débits de l'ordre de $20 \mathrm{mrad} / \mathrm{h}$ alors que l'activité ambiante correspond à environ $20 \mu \mathrm{rad} / \mathrm{h}$. On peut encore voir dans cette anomalie un processus de restauration ou un système de création de centre à plusieurs cibles.

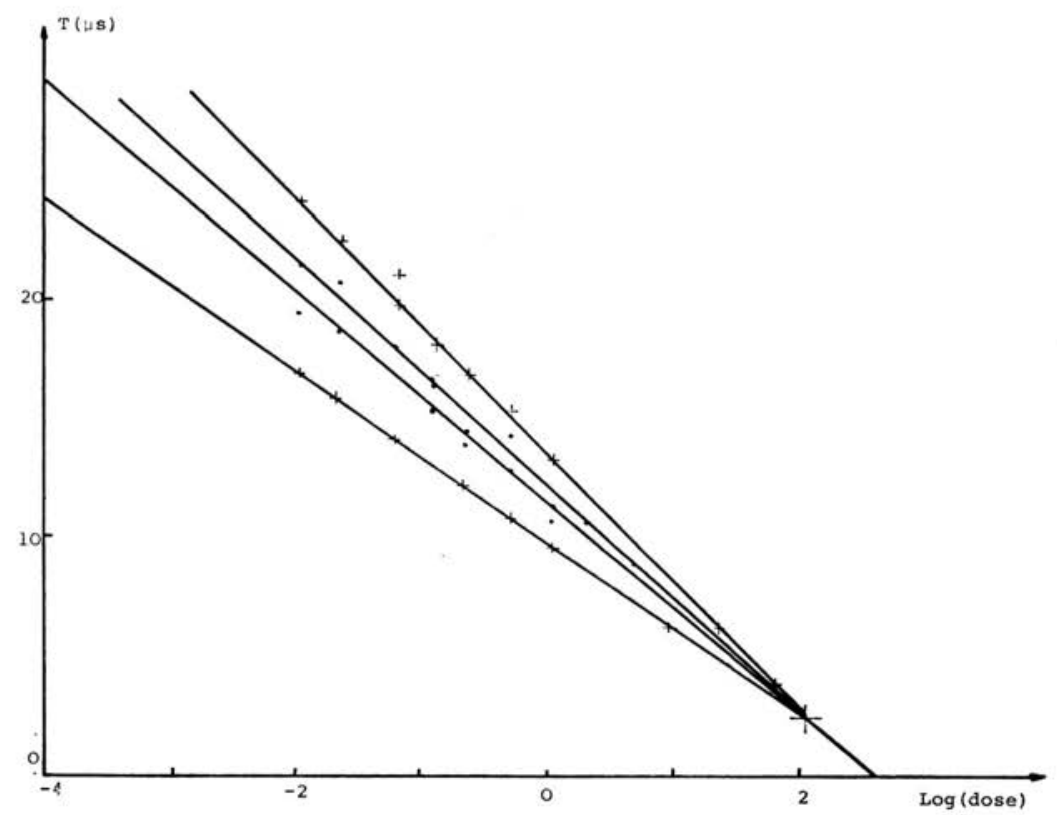

FIg. 11. - Evolution de la constante du domaine 3 pour divers échantillons

La figure 11 présente l'évolution de la constante de temps longue pour divers échantillons dont la fabrication est le résultat de 4 mélanges de base différents. On note ainsi pour la première fois une grande sensibilité de la réponse avec la composition et le passé thermique du verre. 


\section{CONCLUSION}

Le principe de lecture des verres fluorescents fondé non sur une mesure d'intensité, mais sur une étude de 1a forme des courbes de déclin a permis de mesurer la réponse de verres très faiblement irradiés $(100 \mu \mathrm{rad})$ et d'établir une relation simple entre la constante de temps du domaine (4-9 $\mu \mathrm{s})$ et la dose, relation valable de $10^{-4}$ à 50 rads.

La faible prédose «apparente» de ces verres (quelques $\mu \mathrm{rad}$ ) pose le problème de leur réponse en fonction du débit de dose et, par là même, celui de leur réponse en fonction de la nature de la particule incidente. Dans ce but une étude plus approfondie est en cours, en particulier par la diversification des radiations incidentes $(\gamma, \mathrm{X}, \alpha$, neutrons...).

La grande sensibilité de leur réponse en fonction de leur passé thermique peut être une technique de contrôle de leur fabrication; ces mêmes verres lus de façon classique ne présentent dans leurs mesures aucune dispersion significative.

\section{BIBLIOGRAPHIE}

[1] Delarue R., Carpentier S., François H. et Chapuis A.M. Radioprotection, 2, no 3, 1967, 173.

[2] Kastner J., Eggenberger G. et Longnecker A. I.A.E.A. Sympos. on Solid States Dosimetry S.M.-78-33, Vienne, 1966.

[3] Barthe J. Thèse de $3^{\circ}$ Cycle, $\mathrm{n}^{\circ}$ 791, Toulouse, 1969.

[4] Bouvard G. Thèse de $3^{\circ}$ Cycle, no 1479, Toulouse 1973.

[5] Curie D. Luminescence Cristalline. Dunod, Paris, 1960, 196.

[6] Barthe J., Blanc D., Commanay L., François H. et Teyssier J.L. Radioprotection, $1969,4, \mathrm{n}^{\circ} 2,109$.

[7] Barthe J., Blanc D., Commanay L. et Teyssier J.L. Health Physics, 1970, 18, 573. 